\title{
Persée
}

http://www.persee.fr

\section{«Les traditions risquent-elles d'être contaminées »? Paradigmes scientifiques et orthodoxie religieuse dans les cultes de possession au Brésil}

\author{
Véronique Boyer-araujo \\ Journal de la Société des Américanistes, Année 1993, Volume 79, Numéro 1 \\ p. $67-90$
}

Voir l'article en ligne

De la fin du e siècle aux années 1970, le présupposé d'une continuité entre l'Afrique et le Brésil apparaît comme un paradigme dans l'étude des cultes de possession dits afro-brésiliens. Marqués tout d'abord par l'évolutionnisme puis par le culturalisme, les chercheurs - même ceux qui ont critiqué cette dernière école — ont en effet privilégié l'étude des « survivances » africaines et délaissé l'analyse des cultes de possession présentant des traits syncrétiques. La valorisation de certaines " traditions ", en vertu de leur fidélité et de leur immobilisme supposés face à la tentation du mélange, a conduit, dans les études « classiques ", à l'omission systématique du contexte sociologique où s'insèrent les groupes religieux.

\section{Avertissement}

L'éditeur du site «PERSEE » - le Ministère de la jeunesse, de l'éducation nationale et de la recherche, Direction de l'enseignement supérieur, Sous-direction des bibliothèques et de la documentation - détient la propriété intellectuelle et les droits d'exploitation. A ce titre il est titulaire des droits d'auteur et du droit sui generis du producteur de bases de données sur ce site conformément à la loi n $98-536$ du 1 er juillet 1998 relative aux bases de données.

Les oeuvres reproduites sur le site «PERSEE » sont protégées par les dispositions générales du Code de la propriété intellectuelle.

Droits et devoirs des utilisateurs

Pour un usage strictement privé, la simple reproduction du contenu de ce site est libre.

Pour un usage scientifique ou pédagogique, à des fins de recherches, d'enseignement ou de communication excluant toute exploitation commerciale, la reproduction et la communication au public du contenu de ce site sont autorisées, sous réserve que celles-ci servent d'illustration, ne soient pas substantielles et ne soient pas expressément limitées (plans ou photographies). La mention Le Ministère de la jeunesse, de l'éducation nationale et de la recherche, Direction de l'enseignement supérieur, Sous-direction des bibliothèques et de la documentation sur chaque reproduction tirée du site est obligatoire ainsi que le nom de la revue et- lorsqu'ils sont indiqués - le nom de l'auteur et la référence du document reproduit.

Toute autre reproduction ou communication au public, intégrale ou substantielle du contenu de ce site, par quelque procédé que ce soit, de l'éditeur original de l'oeuvre, de l'auteur et de ses ayants droit.

La reproduction et l'exploitation des photographies et des plans, y compris à des fins commerciales, doivent être autorisés par l'éditeur du site, Le Ministère de la jeunesse, de l'éducation nationale et de la recherche, Direction de l'enseignement supérieur, Sous-direction des bibliothèques et de la documentation (voir http://www.sup.adc.education.fr/bib/ ). La source et les crédits devront toujours être mentionnés. 


\title{
« LES TRADITIONS \\ RISQUENT-ELLES D'ÊTRE CONTAMINÉES »? \\ PARADIGMES SCIENTIFIQUES \\ ET ORTHODOXIE RELIGIEUSE \\ DANS LES CULTES DE POSSESSION AU BRESIL
}

\author{
Véronique BOYER-ARAUJO *
}

De la fin du $\mathrm{xIX}^{\mathrm{e}}$ siècle aux années 1970 , le présupposé d'une continuité entre l'Afrique et le Brésil apparaît comme un paradigme dans l'étude des cultes de possession dits afro-brésiliens. Marqués tout d'abord par l'évolutionnisme puis par le culturalisme, les chercheurs - même ceux qui ont critiqué cette dernière école - ont en effet privilégié l'étude des "survivances" africaines et délaissé l'analyse des cultes de possession présentant des traits syncrétiques. La valorisation de certaines "traditions ", en vertu de leur fidélité et de leur immobilisme supposés face à la tentation du mélange, a conduit, dans les études "classiques ", à l'omission systématique du contexte sociologique où s'insèrent les groupes religieux.

Mots clés : Brésil, Afro-brésilien, sociologie religieuse, cultes de possession, revue critique.

\section{¿Las tradiciones corren riesgo de contaminación? Paradigmas cientificos y ortodoxia religiosa en los cultos de posesión brasileros}

Desde fines del siglo XIX hasta los años 1970, el presupesto de una continuidad cultural entre África y Brasil es un paradigma de los estudios sobre cultos de posesión. Primeramente bajo la influencia del evolucionismo y luego del culturalismo, los estudiosos - incluso aquellos que han criticado esta última corriente - privilegiaron el estudio de las supervivencias africanas, abandonando el análisis de los cultos de posesión que presentan rasgos sincréticos. Resulta que la valorización de ciertas "tradiciones", en razón de sus supuestos inmobilismo y fidelidad por oposición a religiones «mezcladas", conduce a la omisión sistemática del contexto sociológico de los grupos religiosos en los estudios " clásicos».

Palabras Claves : Brasil, afro-brasilero, sociologío religiosa, cultos de posesión, reseña crítica.

* 6, passage Lathuile, 75018 Paris.

J.S.A. LXXIX, 1993 : p. 67 à 90. 
Are the traditions in danger of becoming contaminated? Scientific paradigms and religious orthodoxy in the cults of possession in Brazil.

From the end of the XIXth century to nineteen seventies, in the studies of the "Afro-Brazilian" cults of possession, an assumed continuity, between Africa and Brazil was presented as a paradigm. Viewing the subject in the context of concepts derived from cultural evolution and from cultural anthropology, even the researchers who were critical of the latter " school" emphasized the study of "survivals" from Africa to the detriment of syncretics analyses. Given the assumption that certain " traditions" were immuned to assimilation, the "classical" studies systematically ignored the sociological context in which these traditions were enacted.

KEY WORDS : Brazil, Afro-brazilian, sociology of religion, cults of possession, critical review.

A deux moments au moins au cours du $\mathrm{XIX}^{\mathrm{e}}$ siècle les élites du pays se posent de manière aiguë la question de la place et de la valeur à accorder aux noirs brésiliens. Tout d'abord lors de la proclamation de l'indépendance, en 1822, quand les débats s'enflamment sur le thème de l'identité brésilienne. Mais pas plus que l'ancien colonisateur ne saurait incarner dignement la nation nouvellement constituée, le noir, en raison de son statut d'esclave, ne peut représenter l'émancipation récente. En revanche, l'indien mythifié, fier et noble du Romantisme, défenseur des valeurs morales et spirituelles de ce mouvement semble une figure tout à fait adéquate.

Plus tard, l'abolition de l'esclavage en 1888, qui fait en principe des anciens esclaves des citoyens à part entière, relance les discussions sur le devenir de la « race brésilienne ". Bien que, comme l'écrit E. Carneiro (1935:237), il ne reste déjà plus à cette époque que $5 \%$ d'esclaves sur la population totale (les élites avaient compris qu'une main d'œuvre salariée leur serait d'un coût moindre), le changement officiel de statut des noirs dans leur globalité rend les questions plus pressantes : quelle place doivent-ils occuper dans la nouvelle société, de quelles capacités disposent-ils pour contribuer au développement de la nation, et quel impact ont-ils eu sur la culture brésilienne ? ${ }^{1}$.

L'intérêt pour les cultes afro-brésiliens naît de ce que le précurseur $\mathbf{R}$. Nina Rodrigues (1862-1906) appelait " notre problème " le nègre" " (1977: 10). L'étude des Africains au Brésil, pour reprendre l'intitulé de son livre, sera le thème de nombreuses recherches presque toutes réalisées dans des centres urbains de la région nordeste du Brésil durant les trente premières années de ce siècle : à Bahia avec Carneiro, à São Luiz du Maranhão avec Costa Eduardo et Nunes Pereira, à Recife dans l'état de Pernambuco avec R. Ribeiro. La ville de Salvador de Bahia attire aussi des chercheurs étrangers parmi lesquels Frazier qui publia un article sur la famille "nègre ", $M$. Herskovits et $\mathbf{R}$. Bastide.

Dès leur inauguration, les études concernant le monde noir au Brésil s'orientent vers la description et l'analyse des systèmes religieux présentant apparemment un large éventail de similitudes avec l'Afrique. Ces travaux se rejoignent autour de l'idée force qu'il est possible pour un groupe, originaire d'un autre continent et transplanté dans une nouvelle société, de conserver présents et opérationnels ses propres modèles culturels. Cependant, les cultes de possession brésiliens où se retrouvent les descendants des esclaves africains ne forment à l'évidence pas un 
ensemble homogène : les appellations sont multiples, les rites changeants d'un lieu à l'autre et les mythes souvent partiellement oubliés, "bricolés" ou même abandonnés.

Les anthropologues ont alors pour préoccupation première de montrer que les divers cultes de possession constituent l'expression singulière de différents groupes ethniques appelés «nations». Celles-ci se distinguent les unes « des autres par la manière de jouer du tambour (soit avec les mains, soit avec des baguettes), par la musique, par l'idiome des cantiques, par les vêtements liturgiques, quelquefois par le nom des divinités, et enfin par certains aspects du rituel " (Bastide, 1978b : 15). L'identification de la nation à laquelle un lieu de culte se rattache, en fonction des croyances et des pratiques rituelles observées, serait aussi confirmée par l'origine ethnique des ancêtres des adeptes actuels, ou du moins de ceux des dirigeants. Deux ensembles, qui s'opposent par le degré de perméabilité aux influences locales, vont être déterminés : les Candomblés soudanais soucieux de leur intégrité et les Candomblés bantous réceptifs aux autres courants religieux. Et c'est sur les lieux de culte de ce premier groupe que les anthropologues vont concentrer leurs efforts afin d'accréditer la thèse de l'Afrique au Brésil.

Les conséquences sur les cultes de possession seront nombreuses : cristallisation de certaines pratiques religieuses, rejet des lieux de culte jugés non conformes, etc. B. Gois Dantas a mis en évidence l'effet d'occultation des rapports de pouvoir entraîné par de telles analyses. En effet, la valorisation de l'Afrique, selon l'auteur,

« qui à d'autres moments a été utilisée par les noirs pour questionner la domination, a aussi été une forme de domestication des cultes [de possession] plus subtile que celle exercée par les appareils répressifs, dans la mesure où elle n'altère pas les relations entre les classes et les groupes, en se constituant ainsi en une idéologie de la pureté africaine pour cacher la domination. " (1982b : 19)

Le postulat selon lequel le noir brésilien est au fond de lui-même africain (et qu'il se doit de le rester s'il veut avoir une place dans cette société) ne le laisse ainsi pas s'affirmer en tant que citoyen de ce pays.

C'est la remarquable constante des études à rechercher des signes de l'Afrique au Brésil, et les étonnantes ressemblances entre les conclusions des chercheurs et les convictions que Rodrigues exprimait dans ses écrits au tout début de ce siècle, que je me propose de souligner dans cet article.

\section{HISTOIRE ET CULTURE}

C'est irrité par le " culte pour l'indien-emblème, l'indien-conventionnel, de pure fantaisie " et en désaccord avec les « protecteurs de la race nègre " obéissant à une "instinctive» sympathie, que Rodrigues, médecin légiste de son état, entend discuter " scientifiquement " de la valeur culturelle de chaque " race " sans se laisser influencer par « les vertus privées de certaines personnes particulières", dans le but de déterminer le "futur et [la] valeur sociale du métis ario-africain au Brésil " (1977: 4-10). 
Selon l'auteur, pour comprendre le métis, produit du mélange entre le Portugais, le noir et l'indien, il faut distinguer le potentiel de civilisation de chacun et procéder à une évaluation séparée des contributions de ces composantes de la population. Dans ce programme, la priorité est donnée à l'étude systématique des noirs, dans ses dimensions biologique et culturelle, avant qu'ils ne se diluent dans la population blanche. Ainsi guidé par une "transcendante question d'hygiène sociale" (1977 : 1), Rodrigues entreprend à Bahia, identifiée comme "l'unique province ou état brésilien où l'étude des nègres africains peut encore se faire fructueusement " (1977: 17), une enquête sur les "survivances " africaines au Brésil, au cours de la dernière décennie du $\mathrm{XIX}^{\mathrm{e}}$ siècle. L'élaboration de ce catalogue le conduit à s'intéresser particulièrement aux domaines de la religion et du folklore qui semblent avoir mieux résisté aux effets dévastateurs de l'esclavage que les structures sociales.

Pendant les trois décennies suivantes, aucun travail ne verra le jour, hormis celui de M. Querino (1851-1923), homme de couleur autodidacte, qui se propose de montrer la valeur des contributions africaines à la société brésilienne. Mais quand A. Ramos reprend le flambeau des études afro-brésiliennes dès les années 1930, l'intérêt pour les cultes de possession ne s'essouffle plus. Bien que se disant disciple de Rodrigues, cet auteur se distance de celui-ci en rejetant le filet des préjugés raciaux dans lequel, comme de nombreux hommes de son temps, s'était pris son maître. L'infériorité du noir n'est plus définitive, elle est la conséquence d'un développement culturel moindre auquel l'éducation pourra sans peine, mais toutefois très lentement, remédier.

Ramos, dans l'introduction de son livre $O$ negro no Brasil (lère édition en 1934 ; $1940: 25$ ), énumère les différentes facettes de l'étude des noirs : historique avec l'identification des «races nègres" importées (la question sera par la suite développée avec le terme "culture "), anthropo-géographique en évaluant les variations des types raciaux en fonction du milieu, ethnologique avec une analyse comparative des éléments culturels dans le pays d'origine et au Brésil, linguistique en recensant les emprunts aux langues africaines, et enfin sociologique en étudiant l'influence des noirs sur la vie sociale brésilienne.

La connaissance de « l'histoire des nègres colons ", c'est-à-dire la détermination de leur provenance, constitue ici la première étape de l'étude des populations noires. Chaque siècle se caractérise par l'introduction massive d'esclaves présumés d'une même origine qui, des principaux ports de débarquement (Bahia, Recife, Rio de Janeiro), sont dirigés vers une région particulière selon le cycle économique prévalant. Ainsi, aux esclaves provenant de Guinée pendant le Xvi ${ }^{\mathrm{e}}$ siècle et destinés aux plantations de canne à sucre de la région nordeste s'ajoutent, à partir du début du $X{ }^{e}{ }^{e}$, ceux d'Angola réputés plus dociles. Au siècle suivant, quand les mines d'or du sud-est commencent à être exploitées, les Minas (Fanti-Ashantis) de la Côte de l'Or (Ghana) et les Nagôs (Yorubas) de la Côte des Esclaves (Togo, Bénin, Nigéria) sont à leur tour introduits (Bastide, $1960: 63$ ). Enfin, suite au traité signé en 1815 entre l'Angleterre et le Portugal interdisant l'importation d'esclaves des régions situées au nord de l'Équateur (cet accord sera on le sait souvent violé), les plantations de café du sud-est, principaux destinataires de la main d'œuvre, reçoivent en majorité des noirs d'Angola et du Mozambique (Contre-côte). 
Ce panorama général n'est pourtant pas complètement fiable en raison de l'imprécision de certains termes. Par exemple, l'aire géographique désignée sous le nom de Guinée a connu d'importantes variations :

«Beltran [1946:112] conclut son étude rigoureuse : "Durant le $\mathrm{xv}^{\mathbf{e}}$ siècle la Guinée se trouvait dans l'actuelle Sénégambie ; au Xvi ${ }^{e}$ siècle elle arrivait jusqu'aux marais [du cap] de Sainte Anne au Sierra Leone; au [siècle] suivant elle avait atteint le Bénin et au $\mathrm{XVIII}^{\mathrm{e}}$ le Gabon " [... Aussi,] ces délimitations d'aires de provenance [...] abandonnent à la spéculation les origines tribales des esclaves importés aux diverses époques" (Ribeiro, 1978 : 16-17).

Dans les textes historiques, la désignation des populations est également floue. Le terme Mina, qui est parfois réservé aux seuls Fanti-Ashantis, est utilisé par ailleurs pour englober "la quasi totalité des esclaves de l'Afrique supraéquatoriale" (Rodrigues, 1977 : 147). C'est que la chasse aux esclaves, au début limitée au littoral, s'est enfoncée progressivement dans les terres. Or, les hommes ramenés dans les villes côtières sont entassés sur les navires négriers en partance pour le Brésil où ils conservent le nom de l'ethnie dominante dans leur port d'embarquement de l'autre côte de l'Atlantique. Les groupes constitués, appelés "nations" au Brésil et censés rassembler des esclaves d'une même origine, sont ainsi la plupart du temps des associations d'éléments humains composites qui ne doivent leur réunion qu'aux guerres et aux accords diplomatiques.

L'étude se heurte en outre à l'absence de matériaux historiques. En effet, "le décret du 14 décembre 1890 promulgué par Rui Barbosa, alors ministre de la Fazenda [des Finances] et la Circulaire n 29 du 13 Mai 1891 " ont ordonné la destruction par le feu de la plupart des documents douaniers, livres de comptes et d'immatriculation des esclaves qui auraient permis de préciser leur nombre et leur provenance (Ramos, $1946: 269$ ).

Les chercheurs espèrent combler les lacunes d'un travail historique mené uniquement à partir des documents disponibles par un examen attentif des "survivances" africaines dans l'Amérique du $\mathrm{xx}^{\mathrm{e}}$ siècle. Ils attendent de la comparaison des "survivances" actuelles avec les sociétés africaines, ou plus exactement comme le rappelle Bastide $(1960: 20)$ avec les « civilisations africaines des $\mathrm{XVII}^{\mathrm{e}}$ et $\mathrm{XVIII}^{\mathrm{e}}$ siècles", qu'elle lève une partie du voile sur l'identité des populations réduites en esclavage.

Le terme " civilisation " utilisé par la majorité des chercheurs mérite que l'on s'y arrête un instant. Pour Ramos, les civilisations constituent "l'ensemble des manifestations culturelles de l'Homme" dont les formes prises dans le temps et dans l'espace sont appelées cultures (1946:38). Civilisation et culture sont ainsi liées par une relation du général au particulier. Bastide, quant à lui, regroupe " civilisation " et "culture " pour les opposer à la "société " en affirmant que les premières peuvent se perpétuer dans de nouveaux systèmes sociaux si elles trouvent en leur sein des " niches", des espaces où se lover et se reproduire (1960:59). Le degré d'indépendance par rapport aux structures sociales définit donc la culture/ civilisation qui, elle, est exprimée par des valeurs et des symboles. Elle est l'essence de la société (oserait-on dire son âme), ce qui est capable de survivre après sa disparition physique. Aussi, il ne s'agira pas de trouver une société africaine en terre brésilienne mais une culture originaire d'Afrique persistant au Brésil. D'un 
point de vue méthodologique, les auteurs rechercheront des similitudes de traits culturels et non pas une analogie des structures sociales.

L'importance des ouvrages consacrés à cette question et portant sur la totalité du continent américain ${ }^{2}$ permet d'affirmer que la comparaison sur une large échelle était pensée comme un point essentiel de la démarche culturaliste. Les influences de chacune des nombreuses civilisations africaines représentées dans le Nouveau Monde devaient en effet être saisies à travers l'observation des variations qui apparaissent avec plus de netteté en partant de grands ensembles que de petites aires géographiques. Dans un second temps, les typologies préalablement construites faciliteraient l'identification des différentes cultures importées dans une région donnée. Une telle méthode était censée ainsi atteindre un niveau de précision inespéré. Pour le Brésil, les principaux groupes d'influence dont on peut retrouver des traces à des degrés différents dans chaque région seraient au nombre de quatre : les "civilisations soudanaises", les "civilisations islamisées", les " civilisations bantoues du groupe angola-congolais » et les "civilisations de la Contre-côte" (Bastide, 1960 : 62).

La démarche admettant également la possibilité d'isoler, dans les sociétés, diverses parties qui formeraient chacune un tout relativement indépendant du reste, il devenait concevable de se pencher spécifiquement sur certaines sphères du social et de les considérer en elles-mêmes. La division proposée par Herskovits (1960:53) a largement été adoptée : la technologie, l'économie, l'organisation sociale, les Non-Kinship Institutions, la religion, la magie, l'art, le folklore, la musique et le langage sont autant de domaines qui pourraient être traités séparément.

Or au fur et à mesure de la mise en place du cadre de référence, les faits semblent corroborer cette approche. Les éléments de certains domaines, en effet, paraissent s'être mieux maintenus au Brésil que d'autres pratiquement disparus : l'empreinte africaine est plus forte dans la religion, la magie, le folklore et le langage. Plus encore, une relation entre ethnies africaines et conservation d'un domaine spécifique est observée, que la notion de " foyer culturel " (Herskovits, 1960 : 14), un noyau central autour duquel s'articulerait à l'origine la vie sociale, permettrait d'expliquer. Lors d'une situation de contact, la résistance culturelle se cristalliserait sur cet aspect dominant et la sélection des éléments conservés s'opérerait en fonction de leur appartenance à celui-ci. C'est pourquoi les sociétés soudanaises organisées autour de la religion l'ont préservée; les sociétés bantoues dont la cohésion repose sur d'autres bases (par exemple l'élevage du bétail pour les pasteurs d'Afrique orientale, écrit Ramos (1942:68)), ont quant à elles perpétué leur magie, leur folklore et leur langage. Une opposition entre les cultures, parmi lesquelles celles de l'Afrique occidentale sont censées être beaucoup plus tenaces que les autres situées au dessous de la ligne de l'Equateur, s'affirmera dès lors avec vigueur. 


\section{LES AFRICAINS AU BRÉSIL}

Rodrigues (1977 : 71-97) et Bastide (1960:112-137) sont les auteurs qui ont le mieux décrit la résistance des noirs bantous à la condition d'esclave. Dès le $\mathrm{XVII}^{\mathrm{e}}$ siècle, des Africains à peine arrivés au Brésil fuyaient des plantations vers l'intérieur des terres où ils fondaient des communautés appelées quilombos ${ }^{3}$. La primauté de la participation bantoue aux quilombos est certes attestee par l'analyse linguistique, mais l'argument définitif validant cette thèse se trouverait ailleurs, dans un manque. Car si les quilombos avaient été le fait de populations soudanaises, " la constitution de cultes yoruba, jêje ou tsi se serait révélée aux expéditionnaires" (Rodrigues, $1977: 89$ ). Bastide insiste également sur l'inexistence d'un culte originaire d'Afrique :

"Les Guinéens ont une mythologie déjà bien constituée et plus résistante par conséquent au christianisme que le vague animisme des Bantous. L'existence d'une église avec des images de saints tend donc à confirmer que Palmares fut un quilombo bantou. " $(1960: 118)$

La présence d'un syncrétisme si précoce, impliquant selon l'auteur une perte d'identité africaine et l'apparition d'institutions et de formes d'organisation sociale nouvelles - ainsi que Bastide le reconnaît lui-même - le conduit paradoxalement à nier toute l'originalité de Palmares, au point de le comparer à « un phénomène de résistance culturelle, de "régression tribale" " (Bastide, $1960: 124-125)$. Pour Bastide, les quilombos étaient bien plus l'expression d'une nostalgie de l'Afrique que sa reconstitution. Pourtant, on aurait pu penser que les quilombos réunissaient les meilleures conditions possibles (relative indépendance économique et politique) pour la conservation d'une Afrique au Brésil :

"la religion africaine a tendu à reconstituer dans le nouvel habitat la communauté villageoise à laquelle elle était liée et comme elle ne l'a pas pu, elle s'est donné d'autres instruments ; elle a sécrété en quelque sorte, comme un animal vivant, sa propre coquille ; elle a suscité des groupes originaux, à la fois semblables et pourtant différents des groupements africains. 》 (Bastide, $1960: 25-26$ )

Un environnement social favorable n'est donc pas suffisant, selon Bastide, à la réussite de la permanence de la civilisation africaine ; il faut à la base une culture propice pour conserver l'identité. En effet, bien que l'auteur constatant les limites de l'analyse culturaliste se propose d'étudier « le contact entre les peuples en termes de cultures totales" (1960:18), son application du concept de "foyer culturel » le conduit à douter de la possibilité d'une continuité africaine hors du religieux, domaine central à ses yeux et qu'il privilégie aux dépens des autres.

Dans l'ensemble, les Soudanais, organisés autour de la religion, semblent disposer d'une culture plus adéquate à la résistance. Cependant, toutes les populations de ce groupe, en particulier les représentants des Haussas islamisées qui suivaient davantage un syncrétisme musulman-fétichiste que le "pur Islamisme de Mahomet ", ne réussirent pas dans leur entreprise de sauvegarde.

Jusqu'à leur écrasement définitif en 1835 et avec leur disparition celle de l'Islam, les Malês, les Africains musulmans, menaient une vie austère en retrait des autres 
esclaves et des hommes libres qu'ils considéraient Infidèles. A plusieurs reprises au tout début du $\mathrm{XIX}^{\mathrm{e}}$ siècle à Bahia, ils se soulevèrent non contre le système esclavagiste lui-même, mais pour s'emparer de la terre et $\mathrm{y}$ faire travailler leurs propres esclaves. Les insurrections des Malês, menées au nom de l'Islam, étaient de véritables guerres saintes. La répression fut implacable : mort, fouet ou travaux forcés pour les esclaves; déportation en Afrique pour les hommes libres.

L'échec des Malês est, pour Bastide, intimement lié à l'option guerrière et aux visées politiques. Et de fait, le but des insurrections, même si elles apparaissaient comme guidées par un sentiment religieux, était la prise du pouvoir. Or, la solution politique et la solution culturelle seraient incompatibles (Bastide, $1960: 69$ ) ; l'une des deux opère toujours au détriment de l'autre car, nous dit-il, la guerre favorise les phénomènes de fusion culturelle : " pour gagner une guerre il faut profiter des leçons de l'expérience, et emprunter au vainqueur ses armes les plus aiguës » (Bastide, $1960: 392$ ).

En outre, l'élément culturel, comme pour les quilombos bantous, a été déterminant dans la défaite des Africains musulmans :

« les noirs musulmans ont apporté au Brésil beaucoup moins l'Islam que tout ce qui s'y était rattaché des cultes antérieurs à son arrivée en Afrique, et, lorsque l'Islam a disparu, ce sont les anciens éléments seuls qui ont subsisté. Le paganisme, un moment recouvert par l'Islam, a fait irruption au Brésil » (Bastide, 1960 : 213)

Dès que les conditions du maintien de l'Islam n'ont plus été réunies, le côté "fétichiste" a repris l'avantage et ses fidèles sont revenus à leurs anciennes pratiques religieuses. Les Nagôs (Yorubas) qui s'étaient joints aux Malês au cours des révoltes vont alors confirmer leur ascendant sur la colonie noire.

Actuellement seules existeraient encore au Brésil les traditions culturelles des Soudanais yorubas et jêjes et celles des Bantous angolais et congolais dont les systèmes religieux seront le principal objet des études jusqu'aux années 1970. Cependant, autant les cultes de possession d'origine bantoue semblent former un bloc informe, autant les références soudanaises sont précises et multiples. Bastide, dans son livre $O$ Candomblé da Bahia où il entend étudier les cultes de possession soudanais, précise " que les Candomblés Nagô, Quetu ${ }^{4}$ et Ijexa sont les plus purs de tous, et seulement eux seront ici étudiés" (1978b : 15). La raison de son choix l'évidente ressemblance avec l'Afrique - implique d'office l'exclusion des Bantous.

Or, l'énumération détaillée des traditions culturelles auxquelles se rattachent les Candomblés ne doit pas masquer une imprécision des termes semblable à celle que l'on avait notée à propos de l'origine des esclaves. En effet, le groupe Nagô, terme dont les Français désignaient tous les noirs de la Côte des Esclaves de langue yoruba (Rodrigues, 1977 : 102), comprend, selon les auteurs, des "nations" différentes : Ketu, Ijexa, Oyo, Obá (Rodrigues ; P. Verger) ; Mina, Yoruba, Ijexa, Ota, Egba, Fon (ce dernier groupe étant généralement inclus parmi les Gêges) (Querino, 1955). Ribeiro (1978: 78), quant à lui, rapproche l'appellation Nagô de Bahia de celle de Ketu à Recife. D'après la classification de Verger et Rodrigues, Bastide aurait mis sur un pied d'égalité deux sous-groupes (Ketu, Ijexa) avec la catégorie (Nagô) dont ils relèvent. Par Nagô, il aurait en fait désigné le sous-groupe Oyo - identification compréhensible puisqu'il s'agit du nom de l'ancienne capitale 
du royaume des Yorubas. Les divergences quant à la définition du terme "Nagô " montrent assez sa flexibilité.

Quoi qu'il en soit, Y. Castro (1981) cite, parmi les facteurs socio-historiques ayant favorisé la perpétuation d'une tradition Nagô au Brésil, l'introduction tardive des esclaves Yorubas (de 1813 à 1851) à l'heure de l'urbanisation et la poursuite jusqu'à la moitié du XIx ${ }^{e}$ siècle des relations commerciales avec la zone yorubaphone établies depuis la fin du Xvirr siècle. De plus, alors que les Bantous étaient envoyés dans les plantations, ceux que l'on nommera Nagôs étaient concentrés dans les villes du nord-est oủ les activités qu'ils exerçaient (vente de marchandises ou de leur force musculaire dans la rue) leur permettaient de disposer de davantage de temps et de liberté d'action, leur offrant donc de multiples occasions de se réunir. En outre, n'ayant pas, ou peu, bénéficié des chartes de libération dont l'indice de concession fut à son sommet de 1830 à 1850, ils demeurèrent soumis aux mêmes contraintes et modalités d'intégration sociale.

Dans le même article, Castro remarque aussi que le yoruba parlé de nos jours dans les terreiros, les lieux de culte, "se limite à quelques phrases, termes et expressions " :

"Cette supposée "langue Nagô " parlée dans les Candomblés n'est rien de plus qu'une terminologie opérationnelle, spécifique des cérémonies religieuses et rituelles qui se déroulent dans les contextes sacrés, et qui s'appuie sur un système lexical de différentes langues africaines qui furent parlées au Brésil pendant [la période de] l'esclavage.» (1981:65).

D'ailleurs, utilisé au début par les blancs, comme le note Bastide, pour qualifier les pratiques religieuses des noirs, le mot désignant les cultes, Candomblé, est un terme bantou aujourd'hui complètement intégré au lexique des terreiros, quelle que soit la "nation" dont ils se réclament. La méconnaissance des autres langues africaines par les anthropologues a donc contribué à faire de la "nation" Nagô un synonyme de l'Afrique. Et quelles que soient les nuances ou les critiques que chacun apporta à son étude, le Candomblé dit Nagô s'imposa comme le paradigme de l'Afrique au Brésil.

\section{PANORAMA DES CULTES DE POSSESSION}

A la fin du siècle dernier, Rodrigues ne s'étonnait pas « de la prépondérance acquise au Brésil par la mythologie et le culte des Jêjes et Yorubas, au point que, absorbant tous les autres, prévale ce culte presque comme l'unique forme rituelle organisée de nos nègres fétichistes" car "ils se trouvaient plus avancés dans l'évolution religieuse " $(1977: 215)$. Du nord au sud du Brésil, indépendamment de la diversité des formes régionales et des "origines" africaines, les Candomblés Nagôs, dans leur forme bahianaise, ont marqué de leur empreinte les autres cultes de possession afro-brésiliens.

Les noms des dieux nagôs ont été adoptés en tous lieux, enterrant presque toujours dans l'oubli celui des divinités qui leur correspondent dans la langue des 
autres "nations". Sous les ordres d'Oxalá l'orixá de la création de l'humanité et seigneur du ciel, les principales figures du panthéon représentent des forces de la nature, des activités économiques ou guerrières. En revanche le souvenir des mythes relatant leurs aventures et explicitant leur place dans le système de parenté n'est demeuré vivace que dans les Candomblés soudanais. La figure d'Exu, pourtant controversée, y occupe une position de choix : messager entre les dieux et les hommes, "divinité des débuts ou des ouvertures» (Bastide, 1978b : 181), ce serviteur des orixás, qui traduit dans le langage des dieux les questions des hommes, représenterait «le principe de la dialectique et de l'intercommunication" (Bastide, 1978b : 197).

D'ailleurs, avant de procéder à l'appel des divinités, le rituel nagô débute invariablement par une offrande à Exu afin qu'il daigne solliciter la présence des dieux dans le terreiro ou, selon une autre interprétation, pour qu'il ne perturbe pas le bon déroulement de la cérémonie. Rythmés par les tambours et l'agogô (un instrument de percussion en métal), les chants en l'honneur des orixás se poursuivent jusqu'à la possession des initiées, les Filles de Saint. Les dieux, qui s'identifient par un cri propre à chacun d'entre eux, se retirent alors dans une pièce d'où ils ressortent un peu plus tard parés de vêtements somptueux. Les orixás, dans le Candomblé Nagô, ne parlent pas et ne chantent pas, pas plus qu'ils ne boivent, ni ne fument. En exécutant les pas de danse qui les caractérisent, ils se livrent à l'adoration des fidèles. Après le départ des dieux du corps des initiés, la cérémonie s'achève par des chants d'adieux.

La possession par les divinités est contrôlée par une longue initiation au cours de laquelle les initiées auront à apprendre les chants, les danses, les rythmes des tambours, la cuisine des aliments rituels, la préparation des herbes, la manière de sacrifier les animaux... de leur nation. Les nombreuses étapes de la feitura do santo, comme se nomme l'initiation, ont pour but de fixer l'orixá de la candidate (abiâa) dans sa tête, c'est-à-dire de consolider le lien qui les unit. Quand le devin (babalaô) a déterminé avec exactitude à l'aide des buzios (cauris) - l'une des méthodes de divination - le nom de l'orixá de la prétendante au statut de iaô, celle-ci fabrique le collier aux couleurs de la divinité désignée et le confie au chef du terreiro, le babalorixa (ou la ialorixa si c'est une femme), qui le lavera pour lui donner sa force. Dès lors l'abiã sera soumise aux mêmes interdits que son orixá. Vient ensuite une longue période de réclusion pouvant durer trois semaines de la iaô dans la camarinha, la chambre d'initiation.

L'apprentissage terminé, le crâne de la iaô est rasé et les dessins emblématiques de l'orixá sont peints sur son corps. Le chef de culte verse alors le sang des animaux sacrifiés sur le haut du crâne de l'initiée où une incision a été pratiquée. Finalement, l'orixá de la iaô s'identifie officiellement en donnant son nom à l'occasion d'une cérémonie publique.

Les nouvelles iấ se trouvent tout en bas de la hiérarchie complexe des Candomblés et elles doivent respect à leurs aînés : au babalorixá évidemment et à son second, la ia kekerê, mais aussi à tous ceux qui, même s'ils ne peuvent pas être possédés par leur orixá, sont détenteurs d'une charge dans le terreiro: les ekedi, toujours des femmes, qui aident les Filles de Saint lors de la possession, et les ogans, des individus de sexe masculin, qui ont chacun une fonction particulière, l'un 
s'occupant de l'autel, l'autre des sacrifices, un troisième dirigeant la musique... Il est un deuxième groupe formé par les ogans s'occupant tout spécialement des rapports du terreiro avec l'extérieur. Choisis pour leur mérite personnel et leur position sociale, ils procurent au terreiro les ressources financières nécessaires et le défendent en cas de conflit avec la police ${ }^{5}$.

Le Candomblé Nagô, bien qu'il soit considéré comme le plus fidèle à l'Afrique, n'est pas le seul à posséder des lettres de noblesse. Le Xangô de Recife dans le nordeste et le Batuque de Porto Alegre dans le sud participent également de l'univers africain. $\mathrm{Si}$, du fait d'une influence jêje, le nom de certains orixás change dans le Xangô, et si la croyance en la réincarnation des morts et la possibilité de possession par plusieurs divinités (fait qui contraste avec l'unicité de la possession dans le Candomblé) font ici partie intégrante du Batuque, le système cosmologique est relativement semblable en dépit d'associations couleurs/orixás différentes. Les variations sont surtout notables dans le rituel (les Filles de Saint viennent déjà habillées, l'initiation est abrégée) et dans l'organisation hiérarchique (disparition des sacrificateurs et des devins). Ces simplifications ont, selon Bastide (1960 : 295-298), une cause économique. En effet, la population des Xangôs et des Batuques, plus pauvre que celle des Candomblés, ne peut affronter les coûts élevés des sacrifices et des vêtements. Cependant, déclare l'auteur, "s'il y a condensation rituelle, la séquence cérémonielle reste inchangée " (1967: 134).

La Casa das Minas présente à São Luiz, capitale de l'état du Maranhão, constitue un cas à part, guère représentatif des cultes de possession de la région puisque limitée à un terreiro. La tradition jêje qui soutient ce seul centre cultuel n'a pratiquement pas été influencée par le Candomblé Nagô et à la place des orixás, ce sont les voduns qui dominent. Remarquons rapidement qu'à Bahia aussi la fidélité africaine serait concentrée dans de rares terreiros : celui du Gantois, de l'Axé de Opô Afonja et de l'Engenho Velho.

Moins "africains " mais bien plus nombreux, le Candomblé de Caboclo à Bahia, le Tambor de Mina dans l'état du Maranhão, la Macumba défunte et l'Umbanda dans le sud du Brésil ont été plus ouverts aux influences extérieures. Des esprits d'indiens, les caboclos, se sont joints aux orixás et possèdent les fidèles pour «travailler ", c'est-à-dire pour conseiller et soigner les hommes. La possession par Exu, lequel tend de plus en plus à être confondu avec le diable, est autorisée alors qu'elle était exceptionnelle dans le Candomblé. Enfin, la durée de l'initiation est singulièrement rétrécie quand elle n'est pas tout simplement inexistante.

Tous ces cultes de possession ne se trouvent toutefois pas au même stade d'un syncrétisme qui tend à s'accentuer. Ainsi, bien que la présence dans le Candomblé de Caboclo d'une table de correspondances (des orixás nagôs aux caboclos) soit évidente, le côté africain resterait autonome car les cérémonies destinées aux orixás et celles en l'honneur des caboclos sont séparées formellement, spatialement et temporellement (J. E. Dos Santos, 1984 : 89). Dans le Tambor de Mina, écrit Bastide, le peu d'ordre serait donné par ce qui reste d'africain. Enfin, l'industrialisation et la marginalisation du noir dans le système capitaliste ont donné lieu à la formation de la Macumba dont on ne connait malheureusement pas grandchose $^{6}$.

L'Umbanda, qui a remplacé la Macumba dans le sud et dont l'expansion 
semble sans limite, se situe à l'extrémité du continuum. Produit d'une rationalisation des rites de la Macumba au travers de la grille kardeciste ${ }^{7}$, elle représente une étape avancée du processus syncrétique.

\section{SYNCRÉTISME ET PRAGMATISME}

A première vue, l'éloignement de l'Afrique se traduit par une diminution de la durée de l'initiation. Or la transmission du "secret ${ }^{8}$, chargé "d'une force mystique dangereuse, comme tout ce que l'on donne et qu'il faut neutraliser " (Bastide, 1960 : 347), exige lenteur et prudence dans l'acquisition du savoir. Ainsi, dans le Candomblé, l'intégration de l'individu à la communauté se réalise progressivement au fur et à mesure des étapes initiatiques qui permettent l'intériorisation des normes et des valeurs du groupe religieux. Les cultes retenant un fort contenu africain, en s'appuyant sur une tradition culturelle résistante, tendent alors à former des "communautés axiologiques": parmi les valeurs partagées, les ethnologues soulignent l'importance de la vie publique, l'esprit communautaire, le succès comme conséquence de l'accomplissement des devoirs religieux (Bastide, $1960: 302$ ), la nécessité du don, les dieux comme principe de classification des événements...

Cependant, cette longueur même de l'initiation peut avoir un rôle néfaste pour la mémoire africaine car elle risque, "si le "donneur" meurt trop tôt, de faire tomber dans l'oubli des pans entiers de la tradition" (1960:348). Le Candomblé présente d'ailleurs des changements notables par rapport aux cultes de possession africains : moindre spécialisation du corps sacerdotal, disparition de certaines divinités (seules trente à quarante sont remémorées au Brésil), oubli des mythes, occurences plus fréquentes de la possession et prédominance des femmes parmi les fidèles. Rares sont les terreiros, les lieux de culte, qui ont gardé une mémoire parfaite des rites pratiqués par les ancêtres africains ainsi que des mythes racontés. Il arrive même que dans les terreiros les plus traditionnels et les plus anciens, un dieu du panthéon soit remémoré sous un nom n'appartenant pas à la langue dans laquelle il est évoqué.

Mais pour Bastide qui ne nie pas ces différences même s'il les minimise, ce syncrétisme jêje-nagô ne fait que reproduire ce qui existe déjà de l'autre côté de l'Atlantique. Les emprunts d'une "nation" à l'autre n'empêcheraient pas la continuité, car le mode de sélection des éléments obéirait à une logique africaine, où l'adoption de dieux étrangers ne modifie pas le système de représentations des fidèles, ni la structure rituelle. En dépit des pertes et des métamorphoses, la continuité de cette "Afrique en miniature" qu'est le Candomblé avec le continent noir n'est jamais remise en cause : c'est une Afrique mystique. La fidélité est certes fragmentée en raison, d'une part, de la discontinuité historique des traditions et, d'autre part, de la spécificité du domaine où elle s'exerce, mais elle serait totale.

Il est un autre syncrétisme que nul ne songe à nier : l'influence du catholicisme sur les cultes afro-brésiliens. Chaque orixá est en effet clairement mis en relation avec un saint, lequel est différent selon la région considérée : par exemple Ogun est identifié à saint Antoine à Bahia et à saint Georges à Rio de Janeiro. C'est que, dès 
leur arrivée au Brésil, les Africains auraient recouvert d'un vernis chrétien leurs pratiques rituelles dans le but de vénérer leurs divinités en toute tranquillité. Au cours du temps, les "masques " ont eu tendance à se transformer en un système d'équivalences qui, selon Bastide (1960:365), s'appuie sur des similitudes aussi bien structurelles (rôle d'intercesseurs auprès du Dieu suprême) et culturelles (spécialisation semblable) que sociologiques (rapprochement entre confréries catholiques et nations). La "pureté " africaine dans les Candomblés traditionnels resterait néanmoins intacte lors du contact avec le catholicisme : la table de correspondances entre saints catholiques et orixás n'y serait qu'une simple juxtaposition des croyances, voire une réinterprétation du catholicisme en termes africains, et non une fusion.

En revanche, dans les terreiros bantous, où " les besoins esthétiques des noirs [...] ne sont point contrebalancés ici par le souci de la fidélité " (Bastide, $1960: 381$ ), le syncrétisme, dit "morphologique", opère par identification. Ce syncrétisme fusionnant, mais encore religieux, a de grandes chances de devenir un syncrétisme magique qui procède à l'accumulation des procédés sans les intégrer dans un système structuré. Convenant pourtant de l'existence de "presque autant de formes de syncrétisme qu'il y a de strates du social " (Bastide, $1960: 379$ ), l'auteur voit en dernière analyse dans cette divergence des processus syncrétiques une conséquence de la différence entre les cultures soudanaises et bantoues.

Les terreiros bantous seraient plus sensibles à la multiplication des possibilités de résolution des désirs, tant collectifs qu'individuels, parce que leur fragilité culturelle n'a pas permis à la "mentalité façonnée par les vieilles cultures africaines " $(1967 \mathrm{a}: 215)$ de résister aux influences extérieures. Entre autres points de cette "structure mentale des individus, et, par voie de conséquence, de la collectivité » à laquelle Bastide s'intéresse (1967a : 215), et dite ailleurs « inconscient nègre " $(1950: 248)$, il cite « la recherche passionnée des associations fraternelles ou les actes de communion collectives" $(1967: 215)$. Le " principe de coupure " serait l'élément fondamental favorisant la persistance de la personnalité de base africaine : les domaines politique et économique sont soumis au système de valeurs dominant, au monde moderne et capitaliste, tandis que celui de la religion est régi par les valeurs héritées de l'Afrique.

La qualité des productions intellectuelles découle également d'une fidélité affective à l'Afrique. Ainsi, alors que dans le Candomblé la possession est un facteur d'ajustement social et d'équilibre psychologique, car les "masques" (les orixás) proposés par le système religieux permettent de créer "des formes institutionnalisées de névrose latente " (Bastide, $1972: 178$ ), dans les cultes plus syncrétiques où le contrôle social est faible, elle faciliterait la manifestation de troubles comportementaux. La dissolution du système religieux, la désorganisation des représentations marquent la fin de la religion traditionnelle avec l'avènement de l'« anarchie de l'expression médiumnique » et de la pathologie (Bastide, 1950:251). Une fois encore, la gradation dans la cohésion des groupes coïncide avec le degré et la qualité de l'africanité supposées.

Dans les faubourgs des villes où ils sont davantage l'expression d'une solidarité de couleur que celle du partage d'une tradition, les cultes se seraient "dégradés " à tous les niveaux. Le mélange, fruit d'un " relâchement de la censure et du contrôle 
communautaire ", y est avancé et irréversible. Bastide écrit par exemple du Catimbo, une variante des cultes implantés dans la région nord, qu'il est essentiellement individuel et prolétarien, contrastant avec la brillance et la communion des fêtes du Candomblé qui ont "un caractère social organique " évident (1960 : 244-252). Le sentiment d'identité si cher aux Candomblés qui s'exprime par une volonté consciente de maintien de la pureté des traditions, n'existe pas ; ici, le groupe est une somme d'individualités guidées par le désir de compenser une situation de classe insupportable. Si les noirs de la région ont accepté de se joindre au Catimbo, abandonnant leurs propres pratiques religieuses, c'est en raison de leur origine bantoue.

On ne peut s'empêcher de rapprocher ces propos de ceux tenus à la fin du siècle dernier par Rodrigues dans son étude déjà citée :

«Pour considérable qu'ait été l'importation des nègres de l'Afrique australe, du vaste groupe ethnique des nègres de langue tu ou bantou — et le nombre fut colossal —, la vérité est qu'aucun avantage numérique ne l'emporta sur celui des nègres soudanais, auxquels, en plus de cela, revient incontestablement la primauté pour tous les faits dans lesquels, de la part du nègre, il y eut dans notre histoire une affirmation de son action ou de ses sentiments de race. " $(1977: 20)$

Plus loin, le jugement est irrévocable : parmi les esclaves, poursuit Rodrigues, « la prééminence intellectuelle et sociale revient sans conteste aux nègres soudanais " (1977 : 37). Force est de constater la convergence des conclusions malgré un évident changement de méthode et une approche scientifique des phénomènes religieux par ses successeurs. L'équilibre de la relative égalité numérique à peine affirmée est aussitôt rompu par une action marquée des peuples soudanais, comme si eux seuls avaient tenté de prendre leur destin en main. Le contraste entre Bantous et Soudanais est total : culturel, social et psychologique.

L'Umbanda, qui se veut selon R. Ortiz (1978) la religion nationale, est dans cette perspective une "trahison " totale de l'Afrique, la "forme africaine de la magie blanche " (Bastide, 1960 : 451). Aussi, indépendamment de leurs options méthodologiques et théoriques, les chercheurs se limiteront à l'étude des Candomblés où les " survivances " sont les plus visibles.

Herskovits, qui envisage la continuité avec l'Afrique sur un mode pragmatique, espère, par l'étude comparative des modifications des cultures noires dans le Nouveau Monde en référence avec leur origine africaine, mettre à jour les modalités de l'acculturation. Le cas des noirs serait pour lui un exemple plus aisé pour l'analyse de ces processus, et celui des Brésiliens une variante des phénomènes observés.

Bastide, quant à lui, en cherchant à "découvrir l'Afrique au Brésil ", adopte une démarche plus intimiste. Il s'agit de dévoiler " le château intérieur de l'homme noir " afin de comprendre la mentalité africaine préservée au Brésil. La nécessité de la comparaison africaine est telle qu'elle s'imposera à lui dès les premiers moments de l'enquête, en pénétrant dans le terreiro :

« La première opposition entre sacré et profane est l'opposition entre Afrique et Brésil ; par conséquent, le sacré ne pourra exister à Bahia comme dans les autres villes brésiliennes que dans la mesure où l'Afrique a été auparavant transportée d'un côté à l'autre de l'océan. 
C'est la première consécration dont nous devons nous occuper [...] : l'africanisation de la patrie d'exil, ou, de préférence, du Candomblé comme un morceau de l'Afrique " (1978b : 65).

Cette africanisation est perceptible dans l'extrême ritualisation du Candomblé, dans l'occupation de l'espace du lieu de culte (un «microcosme de la terre ancestrale ") reflétant une géographie religieuse aux normes mythiques, et dans la conception du temps formé de moments hétérogènes séparés par une période de chaos et distincts de la vie "profane" (1978b : 97).

Enfin, poussant à l'extrême la quête de l'Afrique, certains ethnologues, comme Verger, se déplacent de l'autre côté de l'Atlantique pour comparer les systèmes religieux des cultes de possession brésiliens avec ceux du golfe de Guinée :

«Les notes qui vont suivre, portent sur les manifestations des cultes rendus aux Amériques [...] et quelques aspects de ces mêmes manifestations dans leur pays d'origine. [...] Le point de départ de cette étude est la ville de Salvador, capitale de l'état de Bahia, au bord de la Baie de tous les saints au Brésil où certains descendants de ces noirs africains sont restés fidèles aux croyances de leurs ä̈eux et continuent à en célébrer les rites au cours de très belles cérémonies. [...] Une familiarité de plusieurs années au Brésil avec les pratiques religieuses des Nago et des Djèdjè (cultes rendus aux Orisa et aux vodun) avait l'avantage de donner aux recherches faites en Afrique des points de départ et de référence précis relativement peu déformés. " (1957: 9)

L'auteur orientera donc ses recherches africaines par rapport aux éléments culturels « conservés » au Brésil. Double censure pour valider l'identité : au Brésil, trouver l'Afrique; en Afrique, confirmer la fidélité brésilienne. Cette démarche aboutit au paradoxe suivant : les Africains réduits en esclavage auraient perdu leur identité africaine de retour au pays (ces retours eurent lieu dans les premières décennies du $\mathrm{xIX}^{\mathrm{e}}$ siècle) après avoir payé leur lettre d'affranchissement (Verger, 1984 : 310); phénomène que l'auteur explique par la forte pression des églises missionnaires sur les populations.

Mais l'analyse de M. Carneiro da Cunha est à ce propos bien plus convaincante. Dans une étude historique sur le retour des esclaves libérés en Afrique, l'auteur montre que la place de l'étranger fait "partie intégrante de la structure sociale » des sociétés traditionnelles (1985 : 149). Aussi, s'il est incontestable que d'anciens esclaves revenus sur la terre de leurs ancêtres s'affirmaient alors Brésiliens, cela ne signifie en aucun cas qu'ils n'étaient plus Africains. Selon Carneiro da Cunha, le maintien et la manipulation d'une identité séparée se doivent à l'intérêt, pour l'ensemble de la société, de "préserver une distinction " qui, conjuguée à d'autres, permettait "l'action politique et le commerce " (1985: 150). Le catholicisme, dans ce cadre, était un signe de l'identité revendiquée.

\section{L'INFLUENCE DE L'EUVRE DE GILBERTO FREYRE}

Les analyses développées à partir des années 1940 ont été très nettement influencées par l'ouvrage de G. Freyre, Maîtres et esclaves, paru pour la première fois au Brésil en 1946. Auparavant, ce dernier avait déjà commencé son oeuvre de 
réhabilitation du noir en organisant, en 1934, le Premier Congrès Afro-brésilien à Recife, qui fut suivi d'un autre en 1938 à Bahia, et réunissait des historiens, des linguistes, des ethnologues et des médecins. Les actes de ces congrès furent publiés par la suite (Estudos afro-brasileiros, Rio de Janeiro, Ariel editora, 1935 ; $O$ negro no Brasil, Civilização brasileira, 1940).

Comme l'écrit R. Motta, "le livre [Maîtres et esclaves] a laissé une marque fondamentale dans l'étude des religions brésiliennes d'influence africaine et aucun, mais littéralement aucun des savants qui vinrent après lui, Brésiliens, Européens ou Nord-Américains, n'a pu se soustraire à son influence décisive " (1978: VIII) qui se résume à deux points : la prise en compte d'une différence radicale entre race et culture ; la considération d'une similitude entre le catholicisme portugais présentant un fort "résidu païen " et les religions africaines (Freyre, $1946: 436$ ).

Dans son livre Maîtres et esclaves, Freyre insiste sur la contribution de tous, selon ses possibilités, à la formation d'une culture nationale. Il dresse un tableau idyllique de la formation de la société brésilienne où l'esclavage fut plus "bénin » qu'ailleurs et où les qualités des uns et des autres s'entremêlent dans un mélange harmonieux grâce au métissage :

« Hybride dès le début, la société brésilienne est, de toutes celles d'Amérique, celle qui s'est constituée le plus harmonieusement quant aux relations entre les races : dans une ambiance presque de réciprocité culturelle, ce qui a permis aux peuples retardataires de profiter au maximum des valeurs et des expériences de peuples plus avancés, à la civilisation adventice de s'adapter au maximum à la civilisation native et le conquérant au peuple conquis. " (1974: 96-97)

La nation brésilienne, qui aurait reçu la tolérance et la volupté du Portugais, la douceur et la propreté de l'indien, l'exubérance et la fécondité du noir, incarne donc la démocratie raciale par excellence. Une telle vision, qui tend à nier les conflits sociaux et à adoucir la gêne que les élites pourraient éprouver au souvenir des indiens décimés et des esclaves morts dans les plantations et les mines, a été accueillie en son temps chaleureusement. Elle avait aussi l'avantage, en concédant aux minorités la reconnaissance d'un apport culturel, d'étouffer dans l'oeuf d'éventuelles revendications. L'affirmation de Freyre à propos des indiens (" ce qui est sauvé, c'est ce que l'on pourrait appeler la partie féminine de la civilisation indigène " disait-il (1974: 177), sous-entendu des aspects secondaires) s'applique également aux noirs : le côté révoltant de l'esclavage est omis au profit de l'ornemental et de l'exotique séduisants.

Les ethnologues ne se contentent cependant pas de la philosophie du métissage de Freyre ${ }^{9}$. Les successeurs de Rodrigues avaient bien évidemment rompu avec les postulats pseudo-scientifiques de celui-ci, mais quelques-unes de leurs remarques semblent parfois se constituer comme un miroir inversé des préjugés de leur prédécesseur ${ }^{10}$ quand il déclarait :

« La race nègre au Brésil, pour meilleurs qu'aient été ses services incontestables à notre civilisation, pour plus justifiées que soient les sympathies qui l'ont entourée [du fait du] révoltant abus de l'esclavage, pour meilleures que se révèlent les généreuses exagérations de ses thuriféraires, constituera toujours un des facteurs de notre infériorité en tant que peuple." (1977: 7) 
Ainsi, le problème insoluble que représente la tache noire pour la nation brésilienne se transforme, chez certains auteurs, en une peur de la contamination de la pureté africaine par les blancs. Dans son ouvrage, Images du Nordeste mystique en noir et blanc, écrit à la suite d'un premier contact avec Bahia en 1948, Bastide affirme :

"Si le Candomblé, à un moment donné, a ouvert la voie à des gestes indécents, la faute en est à la rencontre des races, à l'entrée des blancs qui venaient chercher auprès des Filles de Saint une fièvre des sens, l'excitation que provoquaient en eux l'idée de posséder dans leurs bras, le corps encore tremblant d'un être visité par les puissances surnaturelles. La police, en réglementant les Candomblés, leur a rendu ce service de les faire revenir à leur puritanisme, à leur ancienne pureté. " (1978a : 82) ".

La nocivité du mélange des "races" semble être à son comble puisqu'elle favorise un relâchement des moeurs et une désagrégation des groupes cultuels. Par la suite, dans son introduction au Candomblé da Bahia, publié pour la première fois en 1958, Bastide nuance considérablement sa position :

«La religion du Candomblé, quoiqu'africaine, n'est pas une religion seulement de noirs. Non seulement des mulâtres pénètrent dans le culte, mais aussi des blancs et même des étrangers. Il faut dissocier complètement religion et couleur de peau. Il est possible d'être Africain sans être noir. " (1978b : 12)

Qu'est-il donc arrivé dans cet intervalle pour que, d'une séparation désirée, on en vienne à justifier des exceptions? La réponse est la fascination sans cesse croissante exercée par le Candomblé.

\section{LE CHARME DE L'AFRIQUE DANS LA MODERNITÉ}

Certains chercheurs, envoûtés par le charme et la magie de l'Afrique brésilienne, ont été initiés à la charge d'ogan, les personnes fournissant des aides matérielles aux terreiros. Ils pensaient, et l'idée du principe de coupure devait renforcer leur sentiment, pouvoir abandonner aux portes du lieu de culte leur identité de blanc intellectuel et endosser la panoplie "africaine ". On peut toutefois se demander si aux yeux des fidèles du Candomblé les rapports de classe étaient vraiment annulés, d'autant que bien avant l'arrivée des ethnologues, des représentants des élites blanches fréquentaient et aidaient financièrement les terreiros.

Les cultes syncrétiques comme la Macumba et l'Umbanda, fruits d'un "mélange ", d'une corruption de la pureté originelle, n'ont en revanche jamais eu la faveur des intellectuels. Acceptant les plus diverses influences et intégrant les éléments d'autres traditions culturelles, ils incarnent plus difficilement un univers africain légitime au sein de la société brésilienne. Je citerai, à propos de l'opposition entre le Candomblé et la Macumba construite en fonction du paradigme africain, un passage d'un article de P. Fry, paru en 1984 dans le quotidien $A$ folha de São Paulo (p.9) : 
" Ce que je veux suggérer dans un premier moment, c'est que l'opposition que Bastide construit entre son Candomblé et sa Macumba, exprime, au fond, une autre opposition qui est celle entre le monde moderne, industriel, capitaliste et un monde archaïque et pré-capitaliste. L'Afrique, qu'elle soit l'Afrique du Nigeria ou des Candomblés de Bahia, est ce second monde. Rio de Janeiro ou São Paulo sont le premier, un monde désordonné, individualiste dont les valeurs dominantes sont le lucre, l'intérêt et l'hédonisme. C'est ce monde dionysiaque que Bastide va fuir pour un Candomblé apollinien qui résiste au "ver" de la modernité. De cette façon, le Candomblé représente la possibilité d'existence d'un monde social pré-capitaliste incrusté dans le contexte du Brésil moderne. C'est comme si l'Afrique et le Candomblé représentaient la réalité tangible d'un paradis perdu pour l'Occident, un paradis habité par des bons sauvages. "

Paradoxalement, les blancs qui " abîmeraient » le moins le Candomblé par leur présence appartiennent à l'élite adhérant au discours de la modernité : «C'est comme si blancs et noirs se rencontraient dans la Macumba ou dans le Candomblé en accord avec leurs affinités électives. L'élite blanche rencontre les aristocrates nagôs dans le Candomblé, tandis que la plèbe blanche et mulâtre rencontre les " animistes" bantous dans la Macumba " (Fry, $1984: 8$ ). Au-delà d'une tradition africaine menacée par un capitalisme blanc, s'établissent ainsi des équivalences de position sociale au sein des groupes. La pureté est alors menacée par les exclus du système : métis et blancs pauvres.

Pour garder le plus longtemps possible ce paradis africain en terre brésilienne, cette enclave de tradition au sein de la modernité, il faut l'isoler, empêcher les blancs d'y entrer à moins qu'ils n'acceptent totalement la «mentalité et la civilisation africaines", et, pourquoi pas à l'occasion, rappeler aux fidèles du Candomblé les rites et mythes qu'ils auraient pu oublier. Je prendrai comme exemple de la passion suscitée par ce thème du maintien de l'" africanité " un échange acerbe, paru en 1982 dans Religião e sociedade, entre Verger et Dos Santos, tous deux ethnologues et initiés au Candomblé. Ce que Verger reprochait à Dos Santos "n'était pas tant le fait qu'elle se soit inspirée d'informations erronées ou provenant d'ethnies non-nagô, mais le fait que, pour édifier et "structurer" son oeuvre, elle manipule et modifie les documents cités pour appuyer le système conçu par elle, ce qui est grave et constitue un manque total de probité scientifique ». Il concluait que ses travaux :

" sont un tissu de suppositions et d'hypothèses intelligemment présentées, n'ayant rien à voir avec la culture des Nagô-Yoruba et courant le risque de contaminer les traditions transmises oralement, encore conservées dans les milieux non-érudits. Nous ne sommes plus au temps de Nina Rodrigues, quand les traditions étaient encore assez fortes pour nier et rejeter les extravagances du père Baudin, du lieutenant-colonel Ellis et de compilateurs et intellectuels divers ». (1982:9-10; c'est moi qui souligne)

Verger se pose ici en ardent défenseur de la tradition parce que les noirs eux-mêmes ne sont plus, d'une certaine manière, capables de résister aux transformations, ni d'avoir une position critique par rapport aux nouvelles pratiques. En somme, la tradition africaine n'aurait plus de représentant digne de ce nom au Brésil. La nécessité d'un ethnologue qui comprenne les choses de l'intérieur s'imposerait alors, mais il doit s'agir d'un chercheur responsable, 
qualificatif que Verger semble dénier à Juana E. Dos Santos. Attaquée de la sorte, celle-ci, forte de son "intimité initiatique personnelle », répondit sur le même ton, et non sans fondement :

"L'attaque personnelle de Verger couvre la tentative de contrôler et même d'invalider des divergences idéologiques sous-jacentes. [...] Son insistance sur les coutumes et cultes exotiques semble l'amener à destituer les Africains et leurs descendants du fait d'avoir conscience de leur système de pensée, de posséder une auto-image, des élaborations intellectuelles et des stratégies positives d'action. [...] Cette position semble impliquer non seulement l'impossibilité de transcender le niveau purement descriptif, comme l'incapacité du nègre de transcender l'empirique en réfléchissant sur sa nature profonde. Allant plus loin, il ne sera pas difficile d'arriver par ce chemin à des positions empreintes de racisme. A l'Africain [correspond] seulement l'atavique, le sensoriel, l'hédonisme projeté, à l'Européen [revient] la capacité de raison. De cette façon les idéologues au service de la domination coloniale vident le colonisé de sa conscience d'altérité, de sa capacité à produire la civilisation, et transforment son patrimoine culturel en expressions fragmentaires, désarticulant les signifiants des signifiés. Cette vision folklorisante est l'instrument idéal pour introduire, dans la réalité, des ferments d'aliénation et de cooptation. " (1982:11-13)

Ces deux auteurs se heurtent au fond sur le bien-fondé de la méthode utilisée pour la reconstruction en système des informations recueillies : Verger reproche à Dos Santos d'opérer une rationalisation abusive des croyances; cette dernière condamne sa vision immobiliste d'un système religieux.

C'est donc la capacité du chercheur à rendre compte respectueusement d'un système religieux qui est au coeur de l'échange. La difficulté réside dans la tension induite par la nécessité de rendre compte de l'univers d'un Autre, de son système de représentations et d'actions, à l'aide de nos propres catégories analytiques : " la traduction doit être fidèle et intelligible : fidèle au 'langage de départ'; intelligible dans le 'langage d'arrivée'" (J. Pouillon, 1975 : 158).

Il faut en outre considérer le fait que la société, ou le groupe, n'est pas figée, ni passive. Elle se prononce sur ses conditions d'existence et, en fonction de nécessités internes et externes et de son histoire, construit sa tradition. Ainsi que l'écrit Pouillon (1975 : 159-160) :

«Ce qu'il y a d'inconscient dans une tradition, c'est qu'elle est précisément l'oeuvre du présent qui se cherche une caution dans le passé. "

La tradition est donc un processus dynamique impliquant des choix constants et non pas un catalogue d'éléments présents de toute éternité. P. Boyer insiste également sur cet aspect de la tradition :

"On perçoit communément la tradition comme une sorte de ressassement qui garantit la véracité des énoncés conformes. Or c'est là mettre les phénomènes à l'envers. [...] Contrairement à ce que l'on suppose souvent en anthropologie, la tradition ne peut être conçue comme l'origine des 'croyances' : elle se constitue et se renouvelle perpétuellement par l'accumulation des vérités. " (1986:370)

La tension entre la fidélité à une tradition orale et son explication systématique est, dans l'exemple cité ci-dessus, exacerbée parce que les auteurs participent des deux univers - celui des initiés et celui des ethnologues - dont les démarches ne 
s'accordent pas toujours. En outre, l'usage simultané des deux "langages" complique parfois leur distinction.

Ainsi, si Verger, en tant qu'ethnologue, exprime des craintes pour son objet d'étude - la tradition nagô - Dos Santos, de par sa position dans l'univers des cultes, peut défendre une conception personnelle du système religieux. Mais l'on pourrait tout aussi bien dire que l'attachement du premier à défendre chèrement une version de la tradition témoigne de son identification à l'univers religieux et que l'intérêt de la seconde pour les transformations constitue l'expression de préoccupations scientifiques.

La difficulté de concilier les points de vue (interne-externe) a cantonné pendant longtemps les ethnologues à privilégier une conception culturaliste ou « intimiste " dans l'analyse des cultes de possession, limitant leur étude à l'espace du terreiro.

La délimitation de cet univers africain a exclu la grande majorité des cultes de possession brésiliens. Pourtant, comme le note très justement $\mathrm{S}$. Mintz, il est fort probable que «les manifestations superficielles ne sont pas les seules mesures du degré 'd'africanité' " (1984: 326). Aucun "questionnement sur les survivances blanches " (R. Depestre, 1984 : 264), nulle interrogation sur la fidélité ou la conformité à des traditions européennes, et encore moins indigènes, des structures familiales, du rapport au religieux, des conceptions politiques, etc., n'ont jamais été envisagés par ces auteurs. Tout se passe comme si le Brésil, tenu au bout du compte pour résolument occidental et moderne, ne conservait d'africain que des îlots bien délimités, sans qu'il en soit touché dans son essence.

L'originalité des cultes dits "africains" dans le panorama des religions au Brésil se situe cependant ailleurs que dans une fidélité " objectivement " mesurable. Un premier aspect de leur spécificité réside dans le fait de superposer et confondre l'appellation religieuse avec une origine ethnique supposée. Un second est de distinguer le lieu géographique où les cultes trouveraient leurs racines - l'Afrique — du cadre où ils se développent - la société brésilienne. L'affirmation de la différence du groupe cultuel, qui passe par l'insistance sur la frontière séparant la vie religieuse de l'existence quotidienne reflète cette double proposition. Les notions de rupture et de renaissance, signifiées par l'initiation longue et coûteuse à laquelle doit se soumettre un individu pour s'intégrer à un terreiro, sont d'une importance fondamentale pour la reproduction d'un tel système.

L'idée, qui semblait à première vue s'imposer d'elle-même, d'une communauté soudée et cohérente serait alors un effet des bases explicitées de la constitution de l'identité. Le discours des fidèles ne pourrait donc en aucun cas être pris pour un reflet direct et transparent de la réalité sociale. Evident au regard de la généalogie des terreiros les plus prestigieux, l'aspect conflictuel, relevé entre autres par Carneiro, ne peut être omis même si les luttes pour le pouvoir au sein de ces lieux de culte s'expriment en termes de fidélité à l'Afrique.

L'obligation de se situer par rapport au modèle Nagô a conduit les ethnologues, jusqu'aux années 1970, à concentrer leurs efforts sur l'aspect strictement religieux des cultes de possession, en négligeant l'interférence des catégories religieuses avec le vécu quotidien des fidèles hors des terreiros.

Or est-il fructueux d'isoler des «traits culturels» de l'environnement sociolo- 
gique où ils prennent place, et les "structures mentales" de la société qui les sécrète? Les ethnologues ont volontiers parlé de la fonction d'ajustement psychologique et social des cultes afro-brésiliens, mais ils ont sous-estimé l'importance des structures sociales et délaissé l'étude de la dimension sociologique pourtant recommandée par Ramos. Ce faisant, les auteurs se sont empêchés d'avoir une vision moins statique de la formulation d'une identité culturelle et sociale. Les thèmes des identités personnelles, du processus de transformation et de création religieuses ainsi que celui du rapport entre les groupes sociaux dans le contexte national brésilien, en particulier la relation des terreiros avec les élites ${ }^{12}$ et leur place dans la société, ont, entre autres, été laissés pour longtemps en suspens. *

* Manuscrit reçu en juillet 1992 accepté pour publication en janvier 1993.

\section{NOTES}

1. On le sait, ce sera le début de l'appel à une émigration massive en provenance d'Europe afin de faire disparaître la tache noire en blanchissant la population (Skidmore, 1976).

2. Ramos, As culturas negras no novo mundo, 1946; Herskovits, The negro and the new world, 1960 ; Bastide, Les Amériques noires, 1967.

3. Le quilombo le plus fameux, la République de Palmares, était situé dans l'état d'Alagoas au nordeste du Brésil. Organisé approximativement aux alentours de 1630, il fut totalement détruit en 1697 à la suite d'une expédition de 6.000 hommes venus des états d'Alagoas, de Pernambuco et de São Paulo. Un « roi » administrait cette fédération de villages fortifiés, dispersés sur un vaste territoire, dont la population totale semble avoir atteint, à son apogée, 11.000 hommes. Le surplus de la production, provenant de la chasse, de la pêche et de l'agriculture, était échangé contre des articles manufacturés, surtout des armes et des munitions. Les quilombos n'étaient donc pas complètement isolés : ils entretenaient des relations commerciales avec leurs voisins, et passèrent même un accord avec le gouvernement de Pernambuco en 1678, qui s'empressa d'ailleurs de le violer l'année suivante, leur concédant la jouissance d'une terre en èchange de leur fidélité.

4. On écrit aussi Ketu.

5. Je renvoie au bel ouvrage de Bastide, O candomblé da Bahia (1978b), pour une description détaillée des orixás et des étapes de l'initiation.

6. La seule description nous vient de João do Rio, un journaliste qui fit une série de reportages sur les religions de Rio de Janeiro au début du siècle. Ces textes furent réunis et édités en 1976.

7. Le Kardecisme, du nom de son fondateur français Allan Kardec, de son vrai nom Léon Rivail, est une variante du spiritisme introduite à Rio et à Bahia dès la seconde moitié du XIx siècle (M. Aubrée, 1987 : 14). Les esprits possédant les médiums sont hiérarchiquement classés selon les critères des élites et leur "position spirituelle" tend à se confondre avec le statut de celui qu'ils habitent. Postulant que les âmes se réincarnent en fonction de leurs actions commises en ce bas-monde, les inégalités sociales sont alors perçues comme le reflet des différents niveaux de l'évolution spirituelle.

8. Outre l'aspect strictement religieux du secret, Bastide note qu'il sert d'" arme de défense contre les blancs" (1960 : 335) car l'absence d'explication éviterait les critiques et l'ironie malveillantes. Sans nier l'importance de son rôle mystique, Herskovits $(1960: 18)$ remarque qu'il est pourtant plus facile de recueillir des informations au Brésil qu'en Afrique. Considérer la place de l'ethnologue dans la structure sociale permettrait peut-être de surmonter l'apparente contradiction entre les deux auteurs. Régulièrement désignés comme fauteurs de trouble, ignorants et escrocs par les mass media, les chefs de culte sont méfiants vis-à-vis des étrangers à l'univers des terreiros, et l'ethnologue, dont les ressources économiques sont sans comparaison avec celles des fidèles, est associé aux élites. Cependant, il est un représentant des classes dominantes d'un genre particulier puisque, par son séjour prolongé, il semble témoigner de l'intérêt et, par son obstination à obtenir des informations, une inclination en faveur des cultes de possession. A un premier niveau, répondre à la demande de l'ethnologue est une façon de s'attacher une personne qui jouit d'un prestige certain et de bénéficier d'un appui non négligeable pour affronter la 
concurrence des autres chefs de culte. En second lieu, cet échange place l'ethnologue dans une position d'ogan (même s'il n'en a pas le titre), de protecteur qui a entre autres l'obligation d'aider le terreiro. Les chefs de culte s'ouvriraient ainsi d'autant plus à lui qu'ils en escomptent des retombées immédiates pour la survie du centre et à plus long terme un changement du discours officiel.

9. Freyre ouvrait d'ailleurs la voie à ce changement de perspective : "Par tous ces traits culturels, matériels ou spirituels, les esclaves nègres, des stocks les plus avancés, se révélèrent en condition de concourir, bien mieux que les indiens, à la formation économique et sociale du Brésil. Parfois même, mieux que les Portugais " (1974:265).

10. Ils ne contestent pourtant qu'une partie de ses conclusions, celles qui ont trait aux relations noirs-blancs, tandis qu'au sein du monde noir, la hiérarchie demeure intacte, à savoir la prééminence des groupes soudanais est réaffirmée.

11. Notons que si Rodrigues ( $1977: 246)$ se prononça contre la répression policière, inutile selon lui en raison de l'équivalence entre les formes religieuses et le "stade d'évolution actuel des noirs", et en appela au respect de la Constitution qui assure " la pleine liberté de conscience et de culte" aux Brésiliens, Bastide trouve en celle-ci au moins un aspect bénéfique quant au maintien de la fidélité africaine.

12. Ce point a cependant été brillamment traité par Dantas (1982a, 1982b). Cet auteur montre l'importance de la revendication africaine dans l'élaboration de stratégies d'alliance avec certaines élites.

\section{BIBLIOGRAPHIE}

AubreE, Marion, 1987. - «Entre tradition et modernité, les religions ». Les Temps Modernes, Paris, 491, 142-160.

Bastide, Roger, 1937. - Les équilibres sociaux-religieux. Mémoire destiné au Congrès de l'Institut International de Sociologie, Paris, 107-121.

- 1950. - Sociologie et psychanalyse. Paris, PUF.

- 1959. - "Les métamorphoses du sacré dans les sociétés en transition ". Civilisations, 9 (4), 432-441.

- 1956. - "Immigration et métamorphoses d'un dieu». Cahiers Internationaux de Sociologie. Paris, XX, 45-60.

- 1960. - Les religions afro-brésiliennes : contribution à une sociologie des interpénétrations de civilisations. Paris, PUF.

- 1967a. - Les Amériques noires. Paris, Payot.

- 1967b. - «Le spiritisme au Brésil ». Archives de Sociologie des Religions, Paris, 24, 3-16.

- 1969. - "Le problème des mutations religieuses". Cahiers Internationaux de Sociologie. Paris, 46, 1, 5-16.

- 1970. - "Mémoire collective et sociologie du bricolage". L'Année Sociologique, $3^{\text {e }}$ série, $21,65-108$.

- 1972. - Le rêve, la transe et la folie. Paris, Flammarion.

- 1973. - " Pour une coopération entre la psychanalyse et la sociologie dans l'élaboration d'une théorie des " visions du monde" ". Etudes de Sociologie de la Littérature. Critique Sociologique et Critique Psychanalytique, Editions de l'Institut de Sociologie, Université Libre de Bruxelles, 165-178.

- 1978a. - Images du nordeste mystique en noir et blanc. Paris, Pandora/des sociétés $\left(1^{\mathrm{re}}\right.$ édition 1948).

- 1978b. - O Candomblé da Bahia. São Paulo, Brasiliana (lère édition 1958). 
BoYer, Pascal, 1986. - «Tradition et vérité ». Anthropologie : état des lieux, L'Homme, Paris, 97-98, 347-372.

Carnelro, Edison, sd (1 ${ }^{\text {re }}$ édition 1948). - Candomblés da Bahia. Sl, Ed. de Ouro.

- 1935. - "Situação do negro no Brasil ». In Estudos afro-brasileiros. Rio de Janeiro, Ariel Editora, 237-241.

CARneiro Da Cunha, Manuela, 1985. - Negros, estrangeiros : os escravos libertos e sua volta à Africa. São Paulo, Brasiliense.

CAStro, Yeda Pessoa de, 1981. - "Lingua e Nação de Candomblé ». Africa, São Paulo, 4, 57-77.

Cavalcanti, Maria Laura Viveiros de Castro, 1986. - «Orígens, para que as quero ?». Religião e sociedade, Rio de Janeiro, 13/2, 84-101.

Dantas, Beatriz Gois, 1982a. - Vovô Nagô e papai branco. Campinas, Dissertação de mestrado, UNICAMP.

- 1982b. - « Repensando a pureza nagô ». Religião e Sociedade, Rio de Janeiro, 8, 15-20.

DEPESTRE, René, 1984. - « Bonjour et adieu à la négritude ». L'Afrique en Amérique Latine, Paris, UNESCO, 263-304.

Dos SANTos, Juana Elbein, 1982. - «Pierre Verger e os resíduos coloniais : o outro fragmentado». Religião e Sociedade, Rio de Janeiro, 8, Juillet 11-14.

FreYre, Gilberto, 1978. - Maîtres et esclaves. Paris, Gallimard.

FrY, Peter, 1984. - «Gallus Africanus est, ou, como Roger Bastide se tornou africano no Brasil ». Folha de São Paulo, Folhetim, São Paulo, 15 Juillet, 7-10.

HersKovits, Melville, 1966. - The negro and the new world. Bloomington, Indiana University Press.

Mintz, Sidney W., 1984. - "L'Afrique en Amérique Latine». L'Afrique en Amérique Latine, Paris, UNESCO, 317-332.

MotTa, Roberto, 1978. - Préface. In Ribeiro, Cultos afro-brasileiros do Recife, Recife, MEC-Instituto Joaquim Nabuco de Pesquisas Sociais.

Nunes Pereira, 1979. - A casa das Minas. Petrópolis, Vozes.

OrTIZ, Renato, 1978. - A morte branca do feiticeiro negro. Petrópolis, Vozes.

Poulllon, Jean, 1975. - «Tradition : transmission ou reconstruction?». Fétiches sans fétichisme, Paris, Maspéro, 155-173.

QUERINo, Manuel, 1955. - A raça africana e seus costumes. Salvador, Livraria Progresso Editora.

Ramos, Arthur, 1940. - O negro brasileiro. São Paulo, Companhia Ed. Nacional.

- 1942. - A aculturação negra no Brasil. São Paulo, Companhia Ed. Nacional.

- 1946. - As culturas negras no novo mundo : o negro brasileiro. São Paulo, Companhia Ed. Nacional.

- 1952. - Le métissage au Brésil. Paris, Hermann et Cie Editeurs.

RIBEIRo, René, 1978. - Cultos afro-brasileiros do Recife. Recife, MEC-Instituto Joaquim Nabuco de Pesquisas sociais.

Rıo, João do, 1976. - As religiôes no Rio. Rio de Janeiro, Ed. Nova Aguilar.

Rodrigues, Raimundo Nina, 1977. - Os Africanos no Brasil. São Paulo, Companhia Ed. Nacional. 
SKIDMORE, Thomas E., 1976. - Preto no branco : raça e nacionalidade no pensamento brasileiro. Rio de Janeiro, Paz e Terra.

VERger, Pierre, 1947. - Notes sur le culte des orisa et vodun à Bahia, la Baie de tous les saints, au Brésil et à l'ancienne côte des esclaves en Afrique. Dakar, IFAN.

- 1982. - "Etnografia religiosa ioruba e probidade científica ». Religião e sociedade, Rio de Janeiro, 8, 4-10.

- 1984. - «L'Amérique Latine en Afrique". L'Afrique en Amérique Latine, Paris, UNESCO, 305-315. 